Review Article

\title{
A Review on Gelatin Based Hydrogels for Medical Textile Applications
}

\author{
Seblewongel Petros, ${ }^{1,2}$ Tamrat Tesfaye $\mathbb{D}^{1},{ }^{1}$ and Million Ayele ${ }^{1}$ \\ ${ }^{1}$ Ethiopian Institute of Textile and Fashion Technology, Bahir Dar University, Bahir Dar, Ethiopia \\ ${ }^{2}$ Department of Textile Engineering, College of Engineering, Axum University, Axum, Ethiopia
}

Correspondence should be addressed to Tamrat Tesfaye; tamrat_tsfy@yahoo.com

Received 22 September 2020; Revised 3 December 2020; Accepted 12 December 2020; Published 23 December 2020

Academic Editor: Huining Xiao

Copyright ( 2020 Seblewongel Petros et al. This is an open access article distributed under the Creative Commons Attribution License, which permits unrestricted use, distribution, and reproduction in any medium, provided the original work is properly cited.

\begin{abstract}
Hydrogels are hydrophilic polymer networks that absorb any kind of liquid including biological fluids. Natural polymers and their derivatives along with synthetic polymers are used to form the hydrogels. Networks that constitute the hydrogels are created by the crosslinking of either synthesized polymers starting from monomers or already developed polymers. Crosslinking can be developed either physically if secondary intermolecular forces are involved or chemically in which a covalent bond between polymeric chains is created. Gelatins are natural driven protein polymers. One of the main biopolymers used for producing hydrogels is gelatin. Gelatin has a very wide application other than hydrogels. In this review, hydrogels and their property and synthesis mechanism, as well as their application in biomedical along with gelatin chemistry and application, are reviewed. Due to its nonimmunogenicity, nontoxicity, low cost, and high availability gelatin-based hydrogels could find applications in drug delivery carrier, bioink, transdermal therapy, wound healing, and tissue repair. The beneficiation of gelatin can result in their sustainable conversion into high-value biomaterials on the proviso of the existence or development of cost-effective, sustainable technologies for converting this biopolymer into useful bioproducts.
\end{abstract}

\section{Introduction}

Hydrogels are 3dimensional polymeric units that can take in and keep a huge amount of liquid and other biological fluids by trapping them in their network $[1,2]$. Hence, hydrogels are imparted with hydrophilic functional groups such as $-\mathrm{NH}_{2},-\mathrm{CONH},-\mathrm{COOH}$, and so on; their ability to absorb water is very high [3]. These polymeric units can absorb water from $10-20 \%$ (an arbitrary lower limit) up to thousands of times their dry weight $[4,5]$.

Hydrogels can be made into different physical forms based on the advantage they are intended to be used for such as coatings for implants or catheters, membranes or sheets for a reservoir in a transdermal drug delivery patch and wound dressings, solid moulded forms to be used as soft contact lenses and pressed powder particles to be used as pills or capsules for oral ingestions [2]. The most frequently used synthesize techniques of hydrogel preparation are photo(radical)-initiation system using photoinitiator and crosslinker and the enzymatic crosslinking of functionalized gelatin by which the first one provides good temporal and spatial control on the process [6].

Gelatin is a clear and tasteless protein that has a rheological property of thermoreversible transformation between sol and gel which has been widely utilized in food, pharmaceutical, and photographic industries [7]. Generally, most of the commercial gelatins can be obtained from skin, scale, bones, ligament, and tendon of porcine or bovine [8]. The advantages of using gelatin are its abundance, high carbon content, low price, biodegradability, and nontoxicity [9].

For gelatin-based hydrogels, their rheological properties can be modified by altering the parameters like concentration of photoinitiator and polymer and also the time for exposure to ultraviolet light or the polymerization time and level [10]. In this report, we review the possibilities of using gelatin-based hydrogel in high-value applications. Since 
gelatins are rich sources of proteins and amino acids, we believe that they are a valuable resource; their beneficiation can result in their sustainable conversion into high-value materials and products on the proviso of the existence or development of cost-effective technologies for converting this waste into useful products. The chemical properties, application, and source of gelatin and gelatin-based hydrogels are also reviewed.

\section{Medical Textiles}

The word medical textiles has been interpreted by different scholars at different times. Some of the interpretations are "Medical textiles are products that are used outside the body and are usually not in contact with circulating blood or open wounds; thus, a simple bandage, dressing, eye patch, feminine hygiene product, diaper, an incontinence pad, sling, gauze pad, finger cot, and external casting, brace, and support [11]."

Medical textiles are located at the interfaces between technical disciplines and life sciences. It comprises two different aspects, on the one hand, representing the technical aspect, including textile engineering, chemistry, and testing and certification, and on the other, including the aspects of life sciences like medicine, microbiology, and comfort or strain [12]. Textiles are omnipresent when it comes to the field of human hygiene and medical practice. Advanced medical textiles are developing due to their growth and improvement in the fields of bandaging and pressure garments, wound healing, and controlled-release, implantable devices, medical devices [13].

\section{Hydrogels}

Polymer hydrogels are materials made from natural and synthetic polymers with viscoelastic properties and network structure caused by crosslinker and the solvent, respectively [14]. These polymers are hydrophilic, three-dimensional crosslinked polymer systems capable of absorbing large amounts of water or biological fluids between their polymeric chains to form aqueous semisolid/solid gel networks [5]. Hydrogels could be solid, semisolid, or liquid (Figure 1).

Hydrogels are also known to be divided as gels which are physical or "reversible" if molecular entanglements and/or secondary forces play the principal role in forming the linkage. Physical gels are often rescindable and it is achievable to dissolve them by altering the environmental conditions, such as $\mathrm{pH}$ and the ionic strength of solution or temperature [1]. Examples of reversible hydrogels are "ionotropic" hydrogels formed by the interaction between a polyelectrolyte and an oppositely charged multivalent ion and the polyelectrolyte complexes (complex coacervates) formed by the interaction between two oppositely charged polyelectrolytes [16]. In "permanent" or "chemical" gels, the linkage of covalent bonds linking distinct macromolecular chains can be attained by crosslinking polymers in the dry state or solution. Because the crosslinking of the polymer network prevents the dissolution of hydrogels in water, they swell, which causes a direct increase in their volume [17].

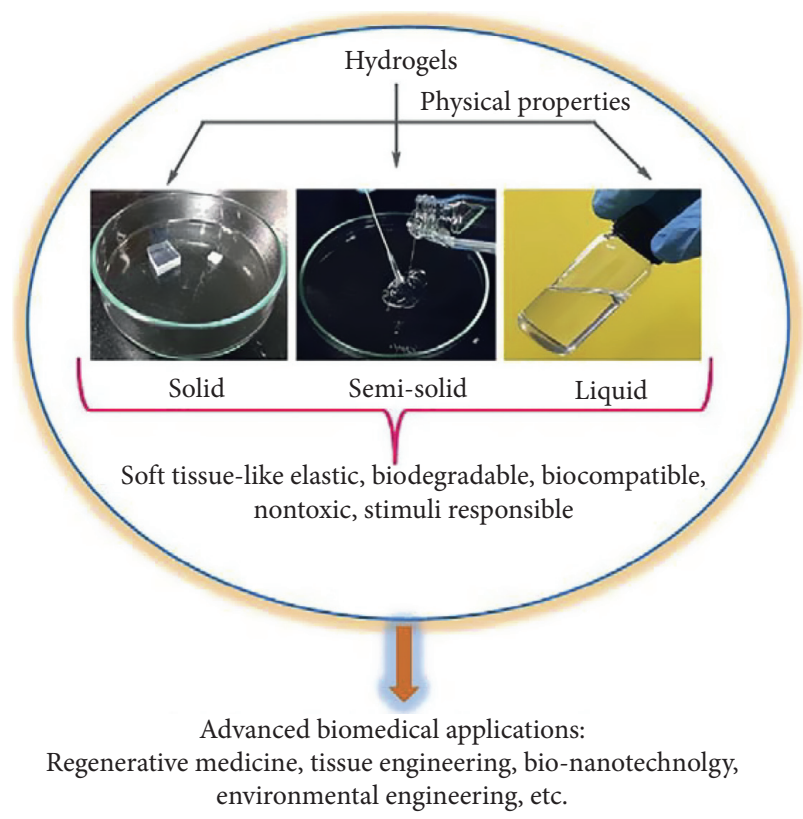

Figure 1: Physical nature of hydrogels (adapted from [15]).

The swelling of a hydrogel is directly affected by waterpolymer interactions, which in turn are affected by the hydrophilicity of polymers: the higher the polymer hydrophilicity, the stronger the water-polymer interactions [18]. The water in a hydrogel can be incorporated as free or bound water. Free water is located at the outermost layer and can be easily removed via mechanical compression or centrifugation. Water attached to the polymer chain is called bound water and forms hydrogen bonds with polar groups of the polymer [19].

Based on the properties and application, hydrogels can be classified according to numerous ways and means [20]. This classification depends on sources of hydrogels, method of preparation, physical and chemical properties, ionic charges, swelling nature, biodegradation rates, and their nature of crosslinking [21]. Because the structure and properties of hydrogels resemble the microenvironment of many human body tissues, they have been broadly used in a diverse range of biomedical applications such as tissue engineering, wound dressing, drug delivery, and so on [22].

\section{Classification of Hydrogels}

Hydrogels can be classified based on different classification bases. The first one is that hydrogels can be classified according to their source which is natural and synthetic [1]. Another classification of hydrogels is based on polymer constituents. Based on this, hydrogels can be homopolymers which are derived from a single species of monomer; copolymers consisted of two or more distinct monomer species with at least one hydrophilic component. Multipolymers, are also called interpenetrating polymeric hydrogel (IPN), an important class of hydrogels, which is made of two independent crosslinked synthetic and/or natural polymer components, confined in a network form. The various classification types are shown in Figure 2. 
Raw materials $\longleftarrow$ Natural and synthetics materials

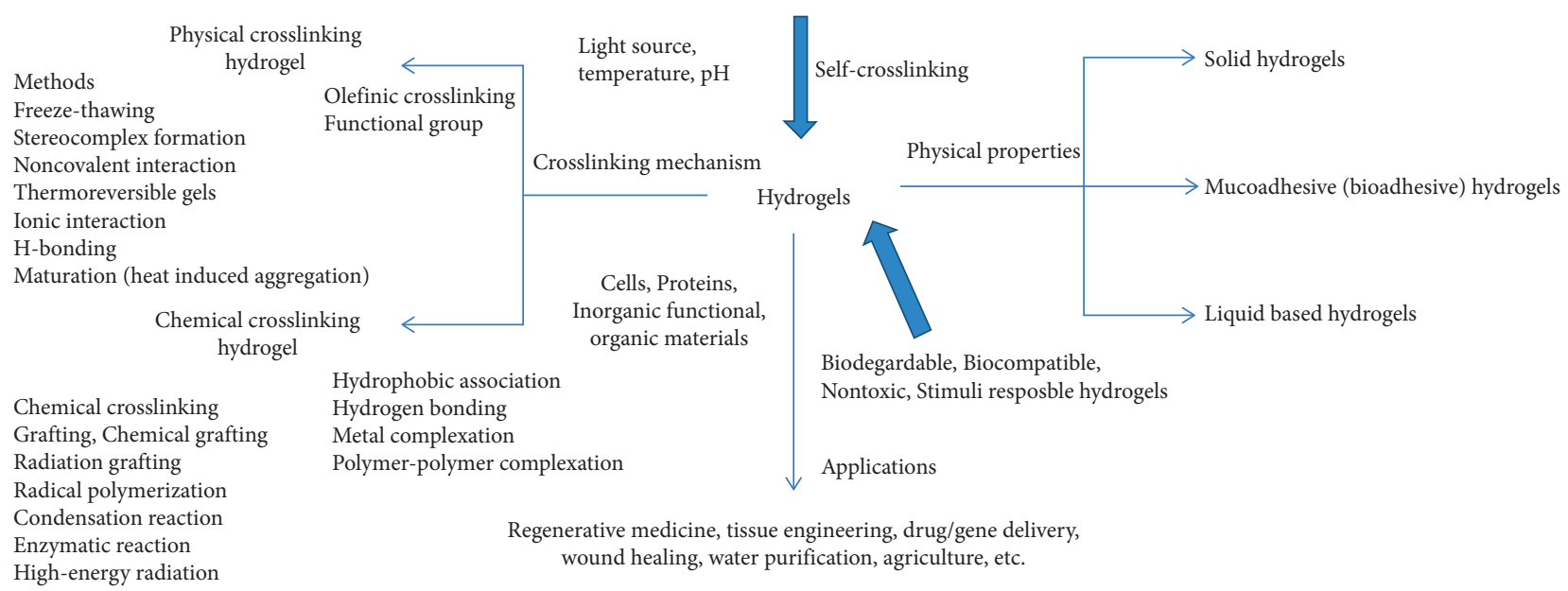

Figure 2: Classification of polymer hydrogels (adapted from [15]).

Another way to classify hydrogels is the cross-linkage created by either it is physical or chemical. "Chemical" or "permanent" hydrogels are categorized based on the presence of covalent bonds within the matrix. These bonds control the degree of swelling of the hydrogel-based on their polymer-water relationship and the degree of reticulation taking place in the matrix. On the other hand, "physical" or "temporary" hydrogels result from ionic bonding, hydrogen bonding, physical interactions, or molecular entanglements, which are reversible by application of force or environmental changes. Hydrogels can also be classified based on their responsiveness to environmental stimuli, which in other words are called "Intelligent" or "smart" hydrogels. The environmental stimulus includes $\mathrm{pH}$, light, temperature, electric fields, solvent composition, pressure, sound, and magnetic fields [2].

\section{Environmental Sensitivity of Hydrogels}

Hydrogels can be stimuli sensitive and respond to the surrounding environment. Smart hydrogel systems with various chemically and structurally responsive moieties exhibit responsiveness to external stimuli including temperature, $\mathrm{pH}$, ionic concentration, light, magnetic fields, electrical fields, and chemicals [2]. These are similar to conventional hydrogels, except these gels may exhibit significant volume changes in response to small changes in these stimuli. The potential response to these stimuli involves changes in shape, volume, phase, and optical properties. The stimuli-responsive volume change of the gels is a result of many factors such as the type of monomers, hydrophilic-hydrophobic balance, crosslink density, osmotic pressure, the conformation of chemical groups, etc. [23].

Temperature-sensitive hydrogels are also called thermals. These stimuli sensitive hydrogels can display changes in their swelling behaviour of the network structure according to the external environments [24]. There are two basic classifications for temperature-sensitive hydrogels; these are negative temperature and positive temperature types of hydrogels. The definition is that the negative temperature is affected when the temperature is below or higher than the lower critical solution temperature, whereas the positive temperature type of hydrogels have reactions towards the temperature ranges in relation to the upper critical solution temperature [14].

$\mathrm{pH}$-sensitive hydrogels are smart hydrogels that consist of a gel structure that varies with a change in $\mathrm{pH}$ values. The $\mathrm{pH}$-sensitive hydrogels expand or contract depending upon the variation which occurs on the $\mathrm{pH}$ of the solutions [25]. The magnetic field is another stimulus for hydrogels; the application of the magnetic field causes a change in the pores of the gel which thereby influences the swelling. The high strength magnetic field can also induce anisotropy in the supramolecular materials, and the relative orientations occur in the network [26]. Electric field-responsive hydrogels are polymers that swell, shrink, or bend in response to an applied electric field. They are usually made of polyanions, polycations, or amphoteric polyelectrolytes [27].

Light-responsive materials can respond to stimuli triggered by the exposition to light sources such as UV, Vis, or infrared. In the case of light-responsive hydrogels, light is a particularly interesting stimulus to manipulate the properties of a hydrogel, as it is a remote stimulus that is easily controlled. Common light-responsive materials are synthesized by the addition of photosensitive groups [28]. Polymers with multiple responsive properties have also been developed elegantly, combining two or more stimuli-responsive mechanisms [29]. This can imply systems that can respond simultaneously and independently to more than one external stimulus, such as $\mathrm{pH}$ and temperatures, have also been developed. An obvious approach would be to copolymerize a temperature-sensitive element with a $\mathrm{pH}$ responsive one as interpenetrating networks [30].

\section{Synthesis Mechanism of Hydrogels}

The process of synthesis in the hydrogel is implying the mechanism used to implement the crosslinking and 
polymerization. Crosslinking is the presence of bonding within the chains of the polymer and intermolecular planar of the molecules used to formulate the hydrogels which prevent the dissolution of the chains before practical use [31]. As a result of the crosslinking of the chains inside the network, the physicochemical properties of the polymers may exhibit changes. These changes will depend on the degree of crosslinking and the degree of crystallinity. Some of the results are the decrease in elasticity, viscosity, and solubility due to the change in the flow property and rheology of the polymer and increase in the glass transition temperature, strength, and toughness of the chains due to the increase in the crosslinked points [20]. Different types of hydrogel synthesis techniques have been applied, such as ultraviolet stereolithographic, gamma radiations, physical, chemical crosslinking, and two-photon polymerization.

6.1. Ultraviolet Stereolithography. Ultraviolet assisted synthesis of hydrogels is a type of solution polymerization in which reaction in the solution is assisted thermally by UV-irradiation. This specific method involves the formation of hydrogels based on photo crosslinking which depends on the presence of photosensitive functional groups. By linking a photosensitive functional group to a polymer, it enables it to form crosslinkages upon irradiation with light such as UV light [5]. Gelatin-based and other natural polymers based hydrogels can be produced using this method of synthesis [10].

Photoinitiators are generally added to improve photosensitivity and to initiate the polymer chains to form bridges or crosslinks. At the same time, the natural polymers and the crosslinker mix is exposed to UV light for the polymerization process. Depending on the absorbency peak of the photoinitiator used, the LED wavelength which will be exposed upon the polymer crosslinker mix will be different. It usually varies from $280 \mathrm{~nm}$ to $400 \mathrm{~nm}$. This method is easily accessible because the instrument can be accessed easily [31].

6.2. Redox Polymerization. Nithusha describes this method of hydrogel synthesis technique as polymerization reactions utilizing redox initiators, and producing free radicals through oxidation-reduction is known as redox polymerization. Redox initiation is also called redox catalysis or redox activation. The main advantages of redox initiators are their lower activation energy of the reaction, which results in a radical formation at a wide range of temperatures, i.e., the initiation reaction occurs relatively at lower temperatures $\left(0-50^{\circ} \mathrm{C}\right)$. Redox initiation has been widely employed in freeradical polymerization. Redox initiators allow the polymerization or the hydrogel formation quickly at very low temperatures [32]. The schematic representation of the synthesis of hydrogels by three-dimensional polymerization is illustrated in Figure 3.

6.3. Gamma Radiation. Radiation processing is a very convenient tool for imparting desirable effects in polymeric materials, and it has been an area of enormous interest in the

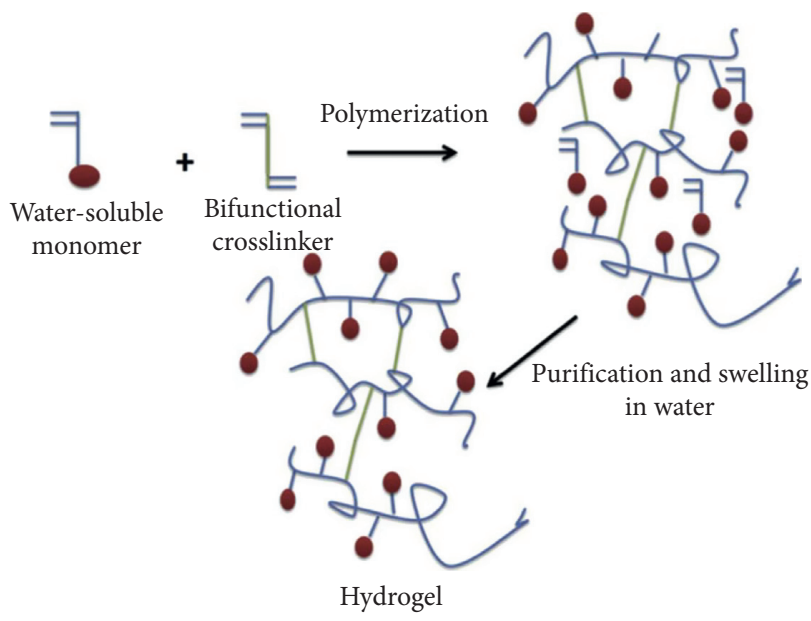

FIGURE 3: Schematic representation of the synthesis of hydrogels by three-dimensional polymerization.

last few decades. The radiation polymerization is a chain reaction in which a large number of chemical changes may follow every single act of ionization or excitation. At present, [10] say radiation-induced copolymerization has been employed for crosslinking the polymeric structures instead of using chemical methods. Since it does not require additives, the generated structures are capable of sterilization and free of carcinogenic materials. Hydrogels that are based on natural polymers have been produced using this synthesis technique for the last decades. In 2014, the synthesis of gelatin-co-PVA copolymer using gamma-irradiation had been studied by Kaur for wound dressing application and had concluded that gamma-ray crosslinked gelatin-PVA hydrogels could be a promising material for tissue engineering applications; it can be useful in delivering drug or nutrient or growth factors directly to the wound site by putting a swab over the hydrogel without removing the hydrogel from the wound site [33]. Figure 4 shows the schematic representation of the mechanism of the synthesis of hydrogels by the gamma-irradiation method.

6.4. Enzymatic. A new approach towards the formation of hydrogels by applying enzyme-catalyzed crosslinking between polymer chains. Different types of enzymes are involved including lysyl oxidase, plasma amine oxidase, and phosphatases, papain, transglutaminase, and peroxidase [5]. The enzymatic crosslinking is a biocompatible approach to enhance the mechanical properties of gelatin hydrogels. Akhtar et al. [31] stated that this method of synthesis had been tried to apply functionalized glutaminyl groups with tetrahydroxy PEG (PEG-Qa). The aqueous solutions of poly(lysine-co-phenylalanine) and the addition of transglutaminase (PEG-Qa) and the addition of transglutaminase resulted in the formation of PEG networks. Transglutaminase catalyzed reaction between the c-carboxamide group of the PEG-Qa and the e-amine group of lysine resulted in the formation of an amide bond [31]. The schematic illustration of enzymatic hydrogel synthesis technique ((a) PEGA and peptide sequence conjugated onto microparticles 


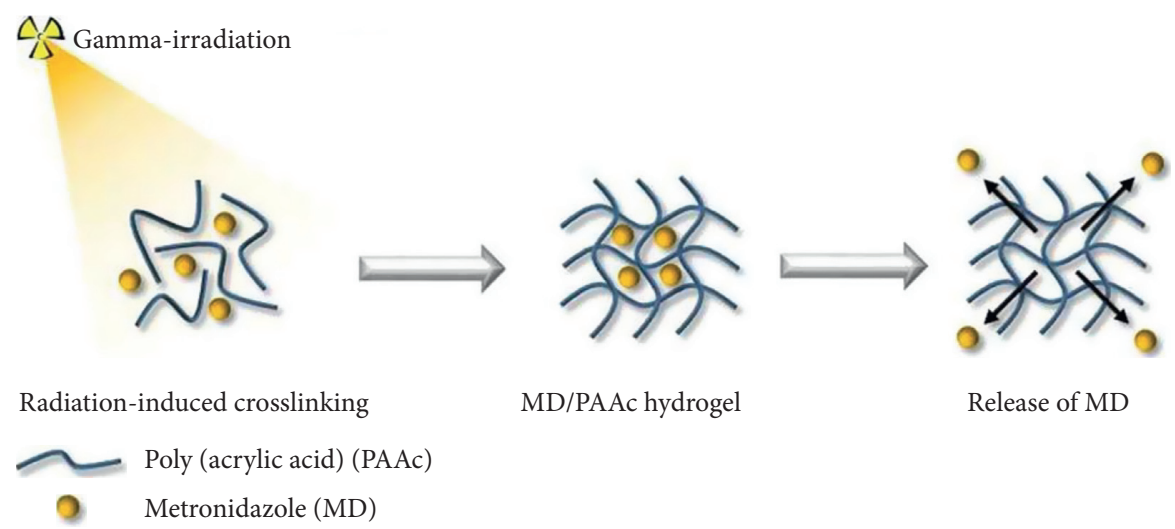

FIGURE 4: Schematic representation of the mechanism of the synthesis of hydrogels by the gamma-irradiation method.

and enzyme cleaved product and (b) PEGA hydrogel) is shown in Figure 5.

6.5. Physical Bonding. Physical crosslinking is referred to as the crosslinking of the polymer chains using physical bonding (Figure 6). For natural polymers, it is possible to just mix the polymer with water to form a gel with hydrogen bonding and other physical bonding [36]. Physical gels can be further classified as weak gels and strong physical gels. The weak physical gels contain the links that are reversible as they are formed from temporary links between chains. These links have the capability of continuously breaking and reforming, and they have finite lifetimes. All of the interactions here in physical crosslinking are reversible and can be disrupted by changes in physical conditions or the application of stress. Also, there is an absence of crosslinking agents and relative ease of production as the macromolecules self-assemble through noncovalent, secondary molecular interactions such as hydrophobic, electrostatic, and H-bonding.

6.6. Interpenetrating Polymer Network (IPN). Figure 7 illustrates a semi- and full interpenetrating polymer network. Interpenetrating polymer network is considered as one of the subcategories of polymer hydrogels. "For example, considering polymers $\mathrm{A}$ and $\mathrm{B}$, in the full IPN, the chains from polymer $A$ are crosslinked with each other, and chains from polymer B are also crosslinked with each other, but crosslinking among chains from polymers A and B is not present. In the semi-IPN, the chains from polymer $\mathrm{A}$ are crosslinked with each other, and chains from polymer B are only physically entrapped therein" [37].

\section{Polymers for Hydrogels}

7.1. Synthetic Polymers. Synthetic polymers are man-made polymers that are derived from petroleum oil. Synthetic polymers are classified into four main categories from application points of view. These are thermosets, thermoplastics, synthetic fibres, and elastomers. Thermoplastics polymers are malleable and moldable at a certain temperature and solidify upon cooling. Likewise, thermoset polymers are similarly hard and cannot change their shape once they are set; as a result of this, they are usually used in adhesives. Synthetic fibres are made to solve problems related to animal fibres and natural plant fibres, and they are made up of a large class of polymers [38]. Synthetic polymers are hydrophobic, mechanically stronger, and chemically strongly bonded and are chemically and mechanically stronger when compared with natural polymers. This improved mechanical strength provides excellent durability to the material by reducing the rate of its degradation.

"Synthetic polymeric hydrogels are generally three-dimensional swollen networks of hydrophilic homopolymers or copolymers covalently or ionically crosslinked". The polymeric hydrogel can be formed by several techniques; but, the most common synthesis method is the polymerization of free-radical vinyl monomers in the presence of swelling agents and bifunctional crosslinking agents. The polymer obtained with this method is exciting in the sense that it shows both solid and liquid-like properties. The polymer formed contains more than $80 \%$ of water. However, it also shows solid-like properties owing to the network formed by the crosslinking reaction, or more like elastic solids in the sense that there exists a remembered reference configuration to which the hydrogel returns after being deformed for a long time [39]. Due to its biocompatible nature, nontoxicity, and high water-absorbing characteristics polyhydroxyethyl methacrylate (PHEM) has been applied in various fields, mainly in the medical industry and PHEM was one of the revolutionary synthetic hydrogel polymers introduced by Laftah et al. [14].

Due to its therapeutic actions in directing cellular functions, such as differentiation and proliferation polyethylene glycol (PEG) is the second most prevalent polymer used for the synthesis of hydrogels in the field of biomedical and pharmaceutical [40]. Polyamide gels are another popular building block of synthetic polymerbased hydrogels. The polymerization occurs by implementing ammonium persulfate and tetramethylethylenediamine as a source of free radicals and as a catalyst, respectively [41]. Polyvinyl alcohol (PVA) hydrogels are synthesized from vinyl alcohol repeating unit which is a synthetic polysaccharide. PVA-based hydrogels have found many applications in medical applications such as wound dressing, implantable devices, contact lenses, and drug delivery devices [42]. 

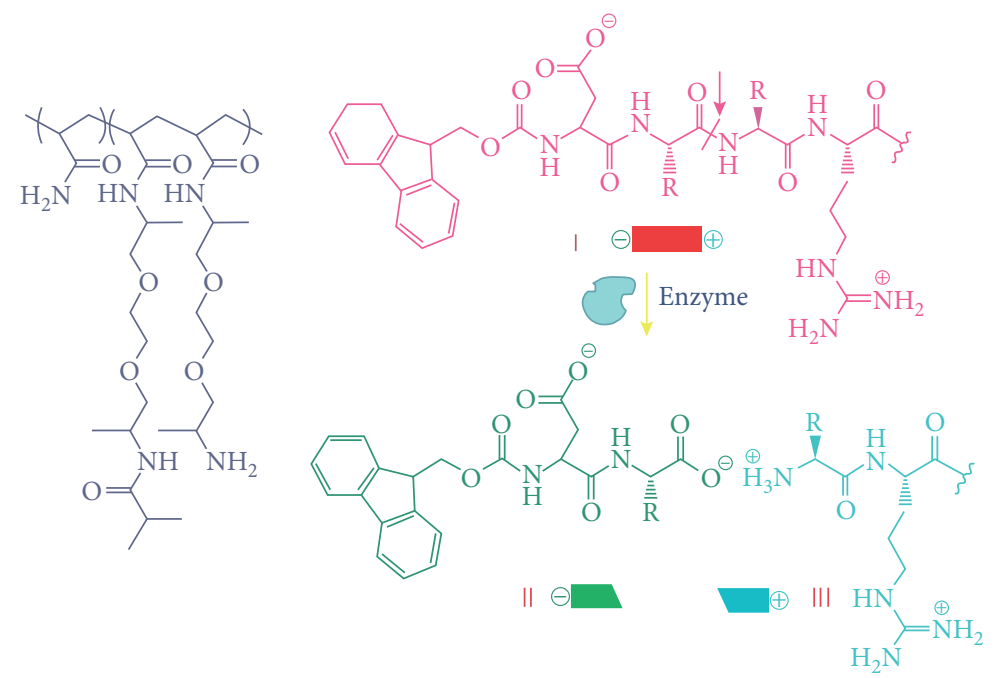

(a)

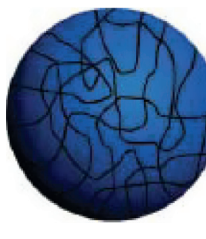

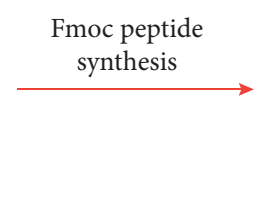

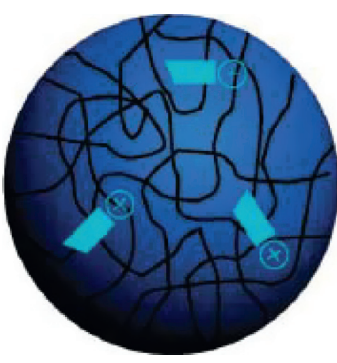

iii

(b)

FIGURE 5: Schematic illustration of enzymatic hydrogel synthesis technique. (a) PEGA and peptide sequence conjugated onto microparticles and enzyme cleaved product and (b) PEGA hydrogel) (adapted from [34]).

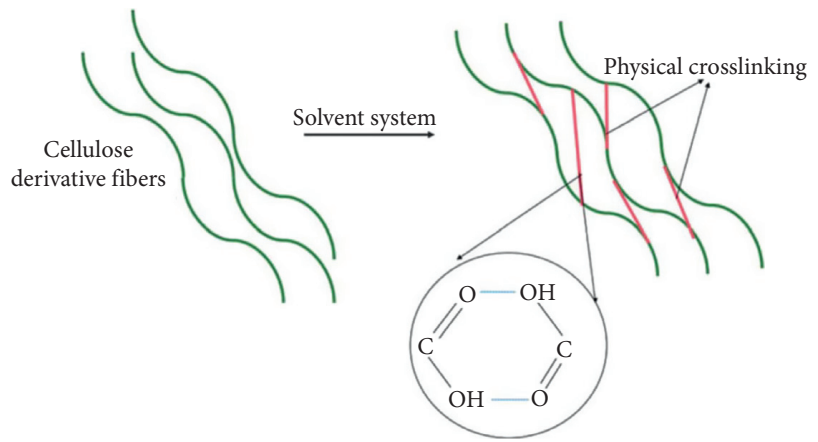

FIGURE 6: Schematic representation of physical hydrogels synthesis technique (adapted from [35]).

7.2. Natural Polymers. Biodegradable polymers have wide applications in a variety of fields such as agriculture, food industry, wastewater treatment, medicine, cosmetics, tissue engineering, drug delivery and wound healing, packaging industry, removal of heavy metals, and so on [43]. One of the main advantages of natural polymers is that they provide hydrogels that have the greatest biocompatibility, as most of them are natural components of the Extracellular Matrix (ECM). Figure 8 illustrates the different types of natural polymers that could be used for the preparation of hydrogel.

7.2.1. Polysaccharides-Based Polymers. Chitosan is considered as a biopolymer containing at least $60 \% \mathrm{D}$-glucosamine waste (D-glucosamine and $\mathrm{N}$-acetyl-D-glucosamine) and it is a deacetylated form of chitin. While this biopolymer is not widely present, it can be easily obtained from the deacetylation of the natural polymer chitin and is considered to be the most abundant biopolymer after cellulose [45]. Chitosan is another natural cationic copolymer that presents well deal of interest for hydrogel structures. Chitosan-based hydrogels are potentially engineering scaffolds to obtain tissue repair achievements [46].

Alginate is a naturally occurring anionic polymer obtained from the extract of brown seaweeds and is produced by two types of bacteria, Azotobacter and Pseudomonas. Due to its ease of availability, biocompatibility, low cost, and chemical composition, alginate hydrogels are ideal for wound healing, tissue engineering application, drug delivery carrier, biofilms, and encapsulation of therapeutic agents. Alginate has been successfully used for the embedding and transport of cells and therapeutic agents and, as a result, is 


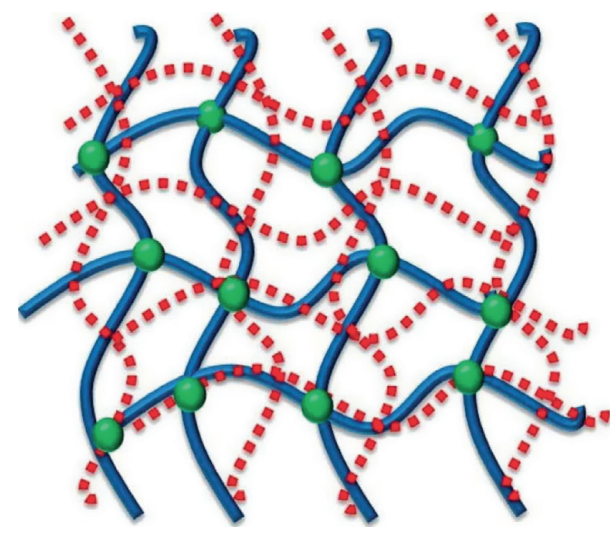

(a)

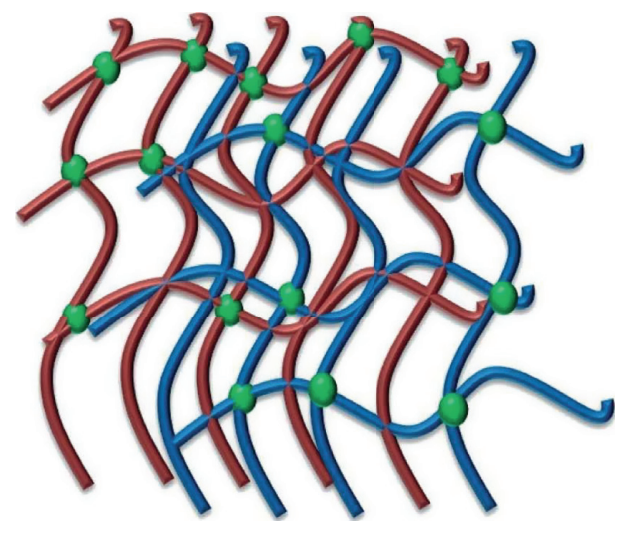

(b)

FIGURE 7: Schematic representation of (a) semi- and (b) full IPN. Blue line: polymer chain A; red line: polymer chain B; green sphere: crosslinked (adapted from [37]).

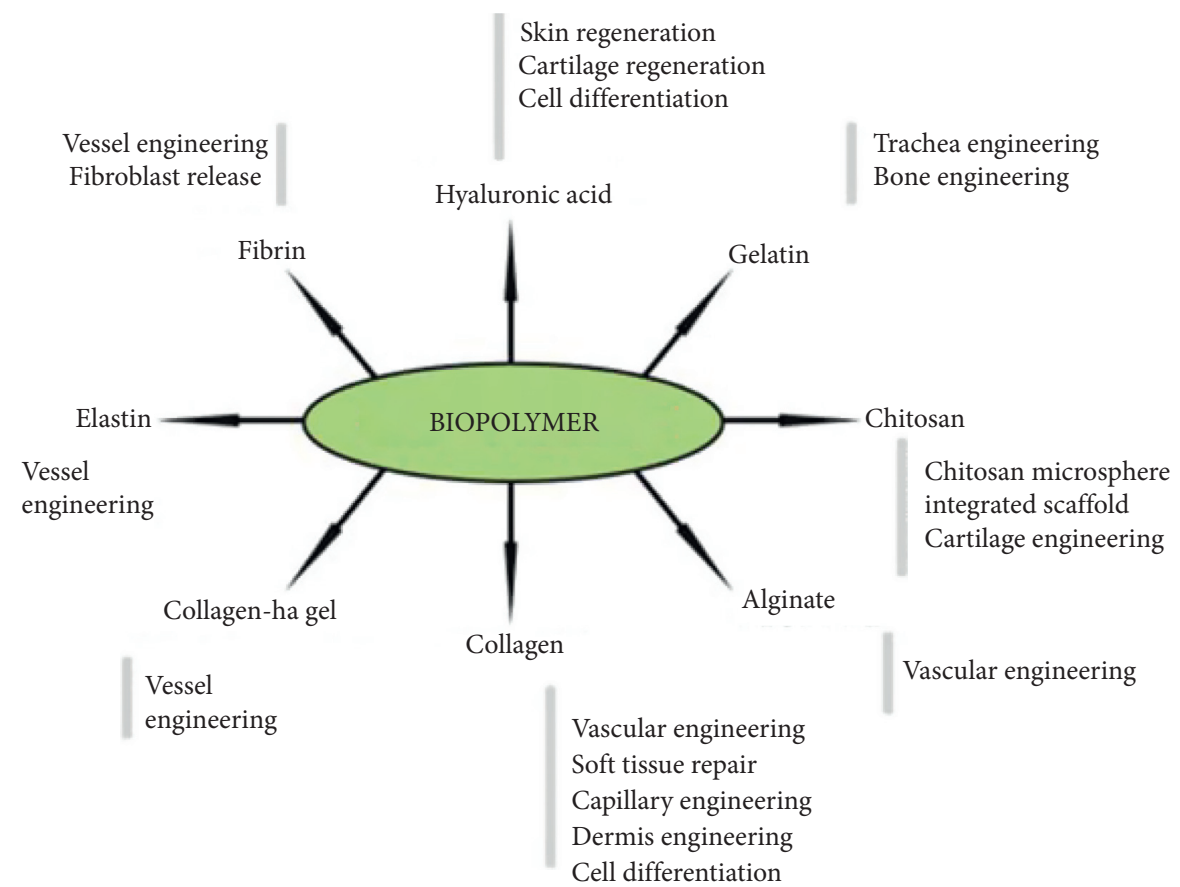

Figure 8: Natural polymers for hydrogel preparation (adapted from [44]).

the most investigated natural polymer for use in the encapsulation of living cells [41].

7.2.2. Protein-Based. Collagen is a natural polymer occurring in natural extracellular matrices (ECM) frequently synthesized from fibroblasts and osteoblasts. The most used form of collagen, collagen type I is obtained from various tissues including skin and ligaments, through the use of enzymatic and acidified processes. Because collagen is obtained from ECM, it is very compatible with biomedical applications [4]. Collagen-based hydrogels are limited in applications because of the mechanical properties and rapid degradability of collagen so improving the properties of this polymer is unquestionable. Improving the properties is done by either a hybrid combination of collagen with other polymers or by altering the crosslinking density [43].

Gelatin is a protein that is obtained from animal sources through thermal denaturation of type I collagen. Gelatin has been a very common protein polymer for producing hydrogels in a different application. Gelatin based hydrogels can be produced in different approaches such as by modifying the amino acid linkages of the polymer, by using crosslinkers to improve the mechanical properties of the polymer, by interpenetrating polymeric network and copolymerization with other natural and synthetic polymers, or the polymer by itself.

Silk is a protein fibrous which has been used in textile production 5000 years ago. Silk has different types according to the source, but the silk from silkworm bombyx mori is the 
popular one. Silk is produced in the process called sericulture, where two types of protein materials are extracted that is fibroin and sericin, where fibroin is used as spun textile material and sericin is usually disposed of [47]. Hydrogels can be formed from reprocessed silkworm silk. A study on the synthesis of hydrogel from silk protein sericin and gelatin was made in a simple and cost-effective fabrication technique without any chemical crosslinkers. The produced sericin-gelatin hydrogel possesses good swelling, mechanical and thermal stability, adequate porosity, and cytocompatibility with poor cell adhesion to use as a wound dressing material [48].

7.2.3. Cellulose-Based Polymer. Cellulose is a common biodegradable polymer found in plants [6]. Cellulose could also be produced by using special bacteria (e.g., Acetobacter xylinum). However, cellulose derived from bacterial microbes $(\mathrm{BC})$ is chemically indistinguishable from plant cellulose (PC), despite their unique and distinct macromolecular structures and physical characteristics. Biological cellulose is purest than plant cellulose because plant cellulose is mixed with other extraneous substances such as lignin, pectin, and hemicellulose [49]. Derivatives of cellulose are produced by acetylation, etherification, and esterification of the hydroxyl group in the cellulose repeating unit. Cellulose and its derivatives are readily biodegradable with bacteria, fungi, and enzymes that are available around the environment, and besides, these are environmentally friendly [50].

\section{Application of Hydrogels}

Hydrogels have a very wide application area (Figure 9). But most importantly, these materials find their importance in biomedical and bioengineering. Medical textiles, which are the basic core of the medicine, can be delivered in most cases by using these hydrogels. Though medicine and health care do need a lot of cautions like biocompatibility, biodegradability, and other factors, still hydrogels, which are natural polymerbased, especially gelatin-based ones, have a lot of significance.

8.1. Wound Dressing. Modern dressings are designed to facilitate wound healing rather than just to cover it. There are different types of advanced wound dressing, and all depend on what they provide for the wound. This classification includes wound dressings that provide protection, wound dressings with antimicrobial functions, wound dressings that provide Autolytic debridement including films, hydrogels, and hydrocolloids, and finally wound dressings which provide chemical debridement [51]. Specific properties of hydrogels such as high surface area, absorbency phenomenon, and variety in product forms are advantageous properties of hydrogels, which makes using them in wound dressing applications desirable [10].

8.2. Contact Lenses. Wichterle and Lim were the first to describe a hydrogel as a synthetic biocompatible material useful for contact lens applications which were based on poly-2-hydroxyethyl methacrylate (PHEMA) in 1960 [51]. The most important advances made on the aspects of contact lenses are the development of silicone-based hydrogels. These provide the property of higher oxygen permeability which protects contact lens wearers especially those who apply them in the night from "induced hypoxia on corneal physiology" [52].

8.3. Drug Delivery Vehicles. Over the past decades, natural polymers (biopolymers) have frequently been used as raw materials for the design of efficient drug delivery systems. Among the different drug delivery systems, hydrogels have particular properties that let them be used as ideal drug delivery systems which is the fact that they are similar to the body's tissues; they have high water content and rubbery consistency [53]. Carrageenan and gelatin-based hydrogels were prepared to apply as a topical drug delivery system. The developed gels were found to be smooth in texture, stable, and hemocompatible in nature. The drug-loaded gels showed sufficient antimicrobial efficacy to be used as a topical antimicrobial gel [54].

8.4. Tissue Engineering. Hydrogels are insoluble hydrophilic polymer networks that are imparted with tissue-like mechanical property and high water content and to make them highly compatible for scaffolds for implantation in empty tubular nerve prosthesis or for direct injection at the lesion site to enhance cell attachment and growth [42].

\section{Gelatin}

9.1. Chemistry and Resource. Gelatin is a natural driven protein polymer that is obtained by the thermal denaturation of collagen and it has widely been applied in industries [7]. It is obtained from many resources such as animal connective tissues, skin, bones, hides, chicken feet, and other collagen-rich parts of animals $[8,55]$. Gelatin from fish sources is another source for gelatin accounting for about $1.5 \%$ of the total gelatin production which is increasing day by day, showcasing the fact that the production of gelatin from alternative nonmammalian sources had grown in importance [56]. Gelatin is a multifunctional ingredient that has long been used in the food industry as a gelling, thickening, and film-forming agent, emulsifier, and stabilizer [57]. Consumption of gelatin worldwide has been reported to be 320,000 tons annually as of 2010 [58].

Type A gelatin is produced by acid processing of collagenous raw material mostly using acidic chemical agents such as hydrochloric acid, sulphuric acid, and so on; type B gelatin is produced by alkaline or lime processing. Because it is obtained from collagen by controlled partial hydrolysis and does not exist in nature, gelatin is classified as a derived protein [59]. Generally, thermal and chemical treatments are involved during the production of gelatin in which three steps are involved; these are raw material preparation, extraction, and finally purification [58]. The typical chemical structure of gelatin is represented in Figure 10. 


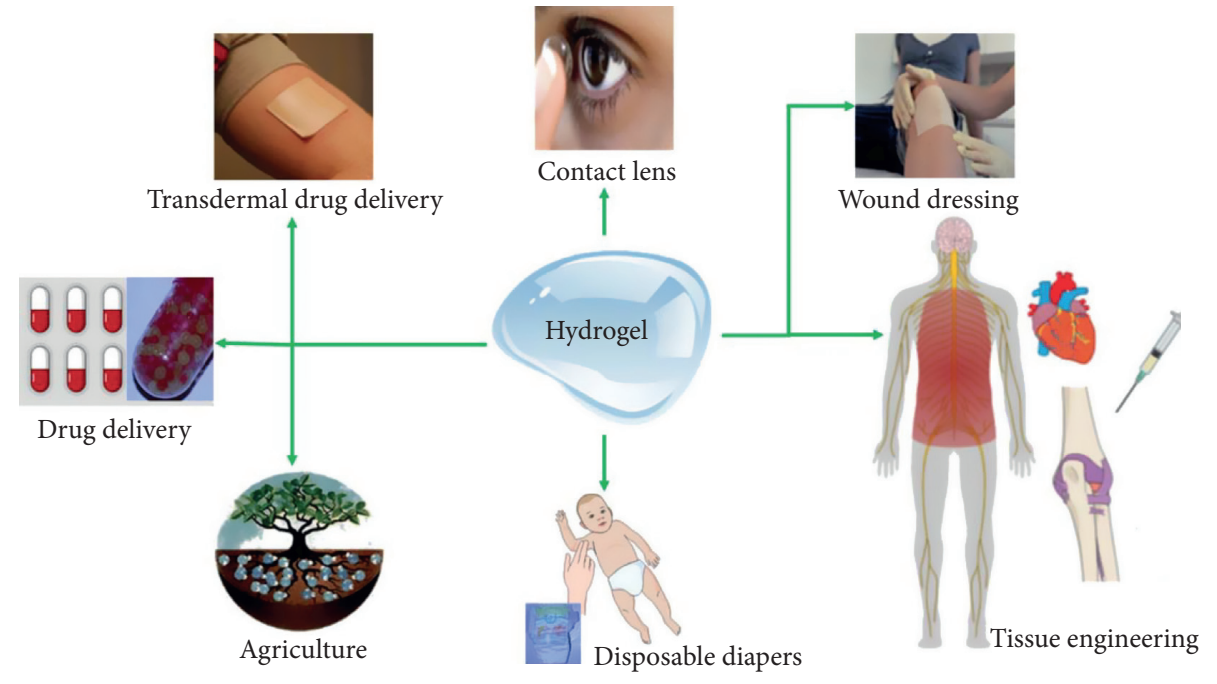

FIGURE 9: Application of hydrogels in various industries.

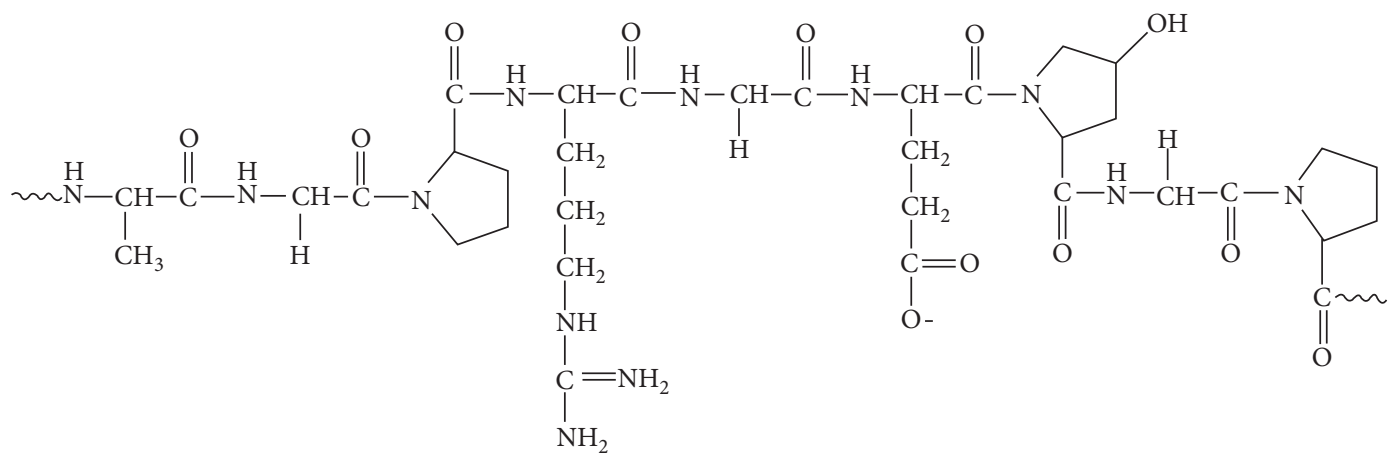

FIgUre 10: Chemical structure of gelatin.

\subsection{Application of Gelatin in the Market}

9.2.1. Food Products. The production of food involves adding many additives and ingredients in order to increase food quality, taste, texture, and colour. Some of the additional ingredients include monosodium Glutamate (MSG), dyes, gelatin, as an example [58]. Many lozenges, wafers, and candy coatings contain up to $1 \%$ gelatin. In these instances, gelatin decreases the dissolution rate. In meat products, such as canned hams, various luncheon meats, corned beef, chicken rolls, jellied beef, and other similar products; gelatin can be used as a clarifying agent in guava juice for obtaining a clear juice by removing the suspended particles $[55,59]$.

9.2.2. Pharmaceutical Applications. In pharmaceutical applications, gelatin has been found in wide ranges of applications such as capsule coats, binding tablets, and sponges for treating wounds and also as a component for vitamin formulations and cosmetics [8]. Capsule production using gelatin involves a combination of gelatin with water or aqueous polyhydric alcohols. Capsules are usually preferred over tablets in administering medicine. Another type of capsules that are elastic or soft is manufactured with a rotary die from two plasticized gelatin sheets which form a sealed capsule around the material being encapsulated [59].

\section{Gelatin Based Hydrogels}

Gelatin-based hydrogels are hydrogels that have the backbone of gelatin as the basic polymeric chains. Hydrogels from protein-based resources are especially useful due to their functional groups. Gelatin-based hydrogels, such as gelatin by itself, combined with other natural and synthetic polymers such as gelatin with alginate, gelatin with chitosan, gelatin with hyaluronan, gelatin with fibrinogen, gelatin with sericin, gelatin with alginate and fibrinogen, and gelatin with alginate, fibrinogen, and hyaluronan, have unique features, such as excellent biocompatibilities, rapid biodegradability, and nonimmunogenicity, in medical applications [60].

These gelatin-based hydrogels are used in the manufacture of contact lenses, matrices for tissue engineering, and drug delivery systems. Developing new uses for gelatinbased hydrogels is another important area of academic research. Gelatin-based hydrogels can be produced chemically modifying the gelatin, or it can be just employing the gelatin with crosslinkers and so on [53]. 
10.1. Application of Gelatin-Based Hydrogels for Medical Textile. Hydrogels formed from hydrophilic components are three-dimensional, porous, and an interpenetrating polymeric network comprising large amounts of biological fluids keeping their structure stable at physiological $\mathrm{pH}$ and temperature. The high affinity of hydrogel for water gives hydrogels a similar physical property with living tissues, such as low interfacial tension and compactness with aqueous media [61]. Due to their effective control over swelling kinetics and high water holding capacity, hydrogels are extremely used in biomedical applications. "The selfhealing and in situ forming hydrogels are very important from a biomedical point of view as it can provide a means for simple, custom-made diagnostics" [62, 63]. Different types of biopolymers could be used as a raw material for hydrogel preparation [64-66].

Gelatin-based hydrogels have stimuli-responsive properties such as thermoresponsive and stimuli-responsive showing the sol-gel transition to the body temperature of humans that is compatible with human tissue. Due to these properties, gelatin based hydrogels have various applications in drug delivery, tissue engineering, and medical textiles [67]. Thermally crosslinked gelatin-based hydrogels can also be used in the manufacturing of composite living fibre, which is made by synthetic polymer as a core material and hydrogel layers having a living cell as a coating material. This new and advanced textile material can be used for the biofabrication of fibrous scaffolds applied in tissue engineering $[64,68]$.

Currently, textile applications of gelatin-based hydrogels are started in drug delivery systems where transdermal drug transport was successfully attained and thermoresponsive gelatin-based hydrogels are effectively forming drug transport systems for textile applications $[69,70]$. Textile-based transdermal therapy with drug-loaded gelatin-based hydrogel is becoming effective in the skincare of human beings [64]. The functionalized textiles coated with gelatinbased hydrogel (stimuli-responsive) can balance a body temperature by controlling the moisture on the skin and provides comfort for the users of smart textile $[67,70]$.

Bioink is the backbone of three-dimensional bioprinting and is composed of a mixture of cells, biomaterials, and bioactive molecules, in a liquid, pregel solution that creates the printed article into a $3 \mathrm{D}$ scaffold or onto a surface. Gelatin-based hydrogels are widely used as bioinks. Methacrylamide modified gelatin (GelMA) has recently gained increasing attention, mainly in the field of biomedical applications [32]. Besides, the gelatin-based hydrogel is used in wound healing function in different forms like patch, gel, ointment, etc. It keeps the wounded area moist and speeds up the healing process by absorbing exudates while maintaining the products of tissue repair, including lysosomes and growth factors in contact with the wounded area [10].

\section{Conclusion}

Medical textiles are textile materials that assist the medicine and healthcare industry. Hydrogels are polymers that are insoluble, crosslinked, hydrophilic, and three-dimensional networks, and they exhibit the ability to absorb a high amount of liquids. Classification of hydrogels can be based on their source, crosslinking, number of polymers, environmental sensitivity, and others which most of them has been reviewed in this paper. Hydrogels that are natural polymers based have a greater advantage of biocompatibility, degradability, and nontoxicity. Gelatin is a natural polymer which is a derivative of collagen and can be sourced from protein-containing part of the animal body including skin, bones, connective tissues, chicken feet, fish scales, and insects. Gelatin based hydrogels have been employed in biomedical and medical textile as gelatin has the property of nonimmunogenicity, nontoxicity, low cost, and high availability. In general, gelatin-based hydrogel could find applications in drug delivery carrier, bioink, transdermal therapy, wound healing, and tissue repair.

\section{Data Availability}

The data will be available upon request.

\section{Conflicts of Interest}

The authors declare that they have no conflicts of interest.

\section{Acknowledgments}

The authors are highly grateful for the financial support received from the Ethiopian Institute of Textile and Fashion Technology at Bahir Dar University, Bahir Dar, Ethiopia.

\section{References}

[1] S. Garg and A. Garg, "Hydrogel: classification, properties, preparation and technical features," Asian Journal of Biomedical and Pharmaceutical Sciences, vol. 2, pp. 163-170, 2016.

[2] K. Saini, "Preparation method, properties and crosslinking of hydrogel: a review," PharmaTutor, vol. 5, pp. 27-36, 2017.

[3] V. M. Gun'ko, I. N. Savina, and S. V. Mikhalovsky, "Properties of water bound in hydrogels," Gels, vol. 3, p. 37, 2017.

[4] Q. Chai, Y. Jiao, and X. Yu, "Hydrogels for biomedical applications: their characteristics and the mechanisms behind them," Gels, vol. 3, no. 1, p. 6, 2017.

[5] R. Parhi, "Cross-linked hydrogel for pharmaceutical applications: a review," Advanced Pharmaceutical Bulletin, vol. 7, no. 4, pp. 515-530, 2017.

[6] S. M. F. Kabir, P. P. Sikdar, B. Haque, M. A. R. Bhuiyan, A. Ali, and M. N. Islam, "Cellulose-based hydrogel materials: chemistry, properties and their prospective applications," Progress in Biomaterials, vol. 7, no. 3, pp. 153-174, 2018.

[7] A. Mariod, S. Abdelwahab, M. Ibrahim, S. Mohan, M. A. Elgadir, and N. Ain, "Preparation and characterization of gelatins from two sudanese edible insects," Journal of Food Science and Engineering, vol. 1, p. 45, 2011.

[8] E. Okonkwo, C. Ezeanyanaso, O. Ofoegbu, E. Ali, and N. Iyamu, "Extraction and characterization of gelatin from cow bones: using substitute demineratizing agent $\left(\mathrm{H}_{2} \mathrm{SO}_{4}\right)$," Global Journal of Pure and Applied Sciences, vol. 13, pp. 189-191, 2007.

[9] M. Ulfa, W. Trisunaryanti, I. I. Falah, and I. Kartini, "Characterization of gelatines extracted from cow bone for 
carbon synthesis," IOSR Journal of Applied Chemistry, vol. 8, pp. 57-63, 2015.

[10] P. Jaipan, A. Nguyen, and R. J. Narayan, "Gelatin-based hydrogels for biomedical applications," MRS Communications, vol. 7, no. 3, pp. 416-426, 2017.

[11] M. W. King, B. S. Gupta, and R. Guidoin, Biotextiles as Medical Implants, Elsevier, Amsterdam, Netherlands, 2017.

[12] V. Bartels, Handbook of Medical Textiles, Elsevier, Amsterdam, Netherlands, 2011.

[13] S. Akter, A. Y. M. A. Azim, and M. A. Al Faruque, "Medical textiles: significance and future prospect in Bangladesh," European Scientific Journal, vol. 10, 2014.

[14] W. A. Laftah, S. Hashim, and A. N. Ibrahim, "Polymer hydrogels: a review," Polymer-Plastics Technology and Engineering, vol. 50, no. 14, pp. 1475-1486, 2011.

[15] K. Varaprasad, G. M. Raghavendra, T. Jayaramudu, M. M. Yallapu, and R. Sadiku, "A mini review on hydrogels classification and recent developments in miscellaneous applications," Materials Science and Engineering: C, vol. 79, pp. 958-971, 2017.

[16] E. S. Dragan, "Design and applications of interpenetrating polymer network hydrogels. a review," Chemical Engineering Journal, vol. 243, no. 572, p. 590, 2014.

[17] J. E. Elliott, M. Macdonald, J. Nie, and C. N. Bowman, "Structure and swelling of poly(acrylic acid) hydrogels: effect of $\mathrm{pH}$, ionic strength, and dilution on the crosslinked polymer structure," Polymer, vol. 45, no. 5, pp. 1503-1510, 2004.

[18] D. Štular, B. Simončič, and B. Tomšič, "Stimuli-responsive hydrogels for textile functionalisation: a review," Tekstilec, vol. 60, no. 2, pp. 76-96, 2017.

[19] F. Ullah, M. B. H. Othman, F. Javed, Z. Ahmad, and H. M. Akil, "Classification, processing and application of hydrogels: a review," Materials Science and Engineering: C, vol. 57, pp. 414-433, 2015.

[20] J. Maitra and V. K. Shukla, "Cross-linking in hydrogels-a review," American Journal of Polymer Science, vol. 4, pp. 25-31, 2014.

[21] S. Kalia, Polymeric Hydrogels as Smart Biomaterials, Springer, Berlin, Germany, 2016.

[22] M. Pulat and G. O. Akalin, "Preparation and characterization of gelatin hydrogel support for immobilization of Candida Rugosalipase," Artificial Cells, Nanomedicine, and Biotechnology, vol. 41, no. 3, pp. 145-151, 2013.

[23] X. Li, W. Wu, and W. Liu, "Synthesis and properties of thermo-responsive guar gum/poly ( $\mathrm{N}$-isopropylacrylamide) interpenetrating polymer network hydrogels," Carbohydrate Polymers, vol. 71, no. 3, pp. 394-402, 2008.

[24] S. K. Gulrez, S. Al-Assaf, and G. O. Phillips, "Hydrogels: methods of preparation, characterisation and applications," Progress in Molecular and Environmental Bioengineering-from Analysis and Modeling to Technology Applications, vol. 117, 150 pages, 2011.

[25] W. Wu, W. Li, L. Q. Wang, K. Tu, and W. Sun, "Synthesis and characterization of $\mathrm{pH}$ - and temperature-sensitive silk sericin/ poly (N-isopropylacrylamide) interpenetrating polymer networks," Polymer International, vol. 55, no. 5, pp. 513-519, 2006.

[26] L.-Y. Chu, R. Xie, X.-J. Ju, and W. Wang, Smart Hydrogel Functional Materials, Springer, Berlin, Germany, 2013.

[27] R. V. Kulkarni and B. Sa, "Electroresponsive polyacrylamidegrafted-xanthan hydrogels for drug delivery," Journal of Bioactive and Compatible Polymers, vol. 24, no. 4, pp. 368384, 2009.
[28] C.-W. Lo, D. Zhu, and H. Jiang, “An infrared-light responsive graphene-oxide incorporated poly ( $\mathrm{N}$-isopropylacrylamide) hydrogel nanocomposite," Soft Matter, vol. 7, no. 12, pp. 5604-5609, 2011.

[29] S. K. Samal, M. Dash, P. Dubruel, and S. Van Vlierberghe, Smart Polymer Hydrogels: Properties, Synthesis and Applications, Smart Polymers and Their Applications, Elsevier, Amsterdam, Netherlands, 2014.

[30] J. F. Mano, "Stimuli-responsive polymeric systems for biomedical applications," Advanced Engineering Materials, vol. 10, no. 6, pp. 515-527, 2008.

[31] M. F. Akhtar, M. Hanif, and N. M. Ranjha, "Methods of synthesis of hydrogels. a review," Saudi Pharmaceutical Journal, vol. 24, no. 5, pp. 554-559, 2016.

[32] K. Nithusha, Development of Gelatin MethacrylamideHydroxypropyl Methacrylate Based Bioink, SCTIMST, Thiruvananthapuram, Kerala, 2017.

[33] I. Kaur, P. Bhati, and S. Sharma, "Radiation induced synthesis of (gelatin-co-PVA)-g-poly (AAc) copolymer as wound dressing material," Advances in Materials Research, vol. 3, no. 4, p. 183, 2014.

[34] P. D. Thornton, R. J. Mart, and R. V. Ulijn, "Enzyme-responsive polymer hydrogel particles for controlled release," Advanced Materials, vol. 19, no. 9, pp. 1252-1256, 2007.

[35] S. Farrukh, K. Mustafa, A. Hussain, and M. Ayoub, Synthesis and Applications of Carbohydrate-Based Hydrogels, Springer, Berlin, Germany, 2018.

[36] M. I. H. Mondal, "Cellulose-Based superabsorbant polymeric hydrogels," in Polymers and Polymeric Composites: A Reference Series, S. Palsule, Ed., Springer, Cham, Switzerland, 2019.

[37] E. C. Frachini and D. F. Petri, "Magneto-responsive hydrogels: preparation, characterization, biotechnological and environmental applications," Journal of the Brazilian Chemical Society, vol. 30, no. 10, pp. 2010-2028, 2019.

[38] I. Gibas and H. Janik, Synthetic Polymer Hydrogels for Biomedical Applications, Wiley, Hoboken, NJ, USA, 2010.

[39] B. D. Ratner and A. S. Hoffman, "Synthetic hydrogels for biomedical applications," Advanced Drug Delivery Reviews, vol. 54, no. 1, pp. 3-12, 1976.

[40] D. A. Gyles, L. D. Castro, J. O. C. Silva, and R. M. RibeiroCosta, "A review of the designs and prominent biomedical advances of natural and synthetic hydrogel formulations," European Polymer Journal, vol. 88, pp. 373-392, 2017.

[41] M. M. Khansari, L. V. Sorokina, P. Mukherjee et al., "Classification of hydrogels based on their source: a review and application in stem cell regulation," JOM, vol. 69, no. 8, pp. 1340-1347, 2017.

[42] E.-E. Hago and X. Li, "Interpenetrating polymer network hydrogels based on gelatin and PVA by biocompatible approaches: synthesis and characterization," Advances in Materials Science and Engineering, vol. 2013, p. 1, Article ID 328763, 2013.

[43] A. K. Mallik, N. Sakib, A. Zaman et al., Benefits of Renewable Hydrogels over Acrylate-And Acrylamide-Based Hydrogels, Springer, Berlin, Germany, 2019.

[44] M. R. Singh, S. Patel, and D. Singh, "Natural polymer-based hydrogels as scaffolds for tissue engineering," in Nanobiomaterials in Soft Tissue Engineering, pp. 231-260, William Andrew Publishing, Norwich, NY, USA, 2016.

[45] H. Liu, C. Wang, C. Li et al., "A functional chitosan-based hydrogel as a wound dressing and drug delivery system in the treatment of wound healing," RSC Advances, vol. 8, no. 14, pp. 7533-7549, 2018. 
[46] F. Ahmadi, Z. Oveisi, S. M. Samani, and Z. Amoozgar, "Chitosan based hydrogels: characteristics and pharmaceutical applications," Research in Pharmaceutical Sciences, vol. 10, p. 1, 2015.

[47] M. Lewin, Handbook of Fiber Chemistry, CRC Press, Boca Raton, FL, USA, 2006

[48] D. M. Nair, "Biodegradable hydrogels from silk sericin \& Gelatin: development and characterization for medical applications," 2015.

[49] A. Sannino, C. Demitri, and M. Madaghiele, "Biodegradable cellulose-based hydrogels: design and applications," Materials, vol. 2, no. 2, pp. 353-373, 2009.

[50] M. I. Mondal and M. O. Haque, Cellulosic Hydrogels: A Greener Solution of Sustainability, Cellulose-Based Superabsorbent Hydrogels, Springer, Cham, Switzerland, 2019.

[51] E. Caló and V. V. Khutoryanskiy, "Biomedical applications of hydrogels: a review of patents and commercial products," European Polymer Journal, vol. 65, pp. 252-267, 2015.

[52] F. Stapleton, S. Stretton, E. Papas, C. Skotnitsky, and D. F. Sweeney, "Silicone hydrogel contact lenses and the ocular surface," The Ocular Surface, vol. 4, no. 1, pp. 24-43, 2006.

[53] A. Bakravi, Y. Ahamadian, H. Hashemi, and H. Namazi, "Synthesis of gelatin-based biodegradable hydrogel nanocomposite and their application as drug delivery agent," Advances in Polymer Technology, vol. 37, no. 7, pp. 2625-2635, 2018.

[54] J. R. Padhi, "Preparation and characterization of novel gelatin and carrageenan based hydrogels for topical delivery," 2015.

[55] R. Widyasari and S. Rawdkuen, "Gelatin from chicken feet: papain-assisted extraction, characterization and its application," Journal of Food Science and Agricultural Technology (JFAT), vol. 1, pp. 136-143, 2015.

[56] T. U. Rashid, S. Sharmeen, S. Biswas et al., Gelatin-Based Hydrogels. Cellulose-Based Superabsorbent Hydrogels, Springer, Cham, Switzerland, 2018.

[57] L. Du, L. Keplová, Z. Khiari, and M. Betti, "Preparation and characterization of gelatin from collagen biomass obtained through a $\mathrm{pH}$-shifting process of mechanically separated Turkey meat," Poultry Science, vol. 93, no. 989, p. 1000, 2014.

[58] A. Bahar, R. Rusijono, and N. Kusumawati, "Extraction and characterization of the base halal gelatin based on bovine bone," in Proceedings of the Seminar Nasional Kimia-National Seminar on Chemistry (SNK 2018), Atlantis Press, Paris, France, January 2018.

[59] M. Sebastian, "Industrial gelatin manufacture-theory and practice," 2014.

[60] X. Wang, Q. Ao, X. Tian et al., "Gelatin-based hydrogels for organ 3D bioprinting," Polymers, vol. 9, no. 12, p. 401, 2017.

[61] O. Wichterle and D. Lím, "Hydrophilic gels for biological use," Nature, vol. 185, no. 4706, pp. 117-118, 1960.

[62] L. A. Sharpe, A. M. Daily, S. D. Horava, and N. A. Peppas, "Therapeutic applications of hydrogels in oral drug delivery," Expert Opinion on Drug Delivery, vol. 11, no. 6, pp. 901-915, 2014.

[63] S. R. Van Tomme, G. Storm, and W. E. Hennink, "In situ gelling hydrogels for pharmaceutical and biomedical applications," International Journal of Pharmaceutics, vol. 355, no. 1-2, pp. 1-18, 2008.

[64] S. Chatterjee, P. Hui, and C.-W. Kan, "Thermoresponsive hydrogels and their biomedical applications: special insight into their applications in textile based transdermal therapy," Polymers, vol. 10, no. 5, p. 480, 2018.
[65] B. Joseph, S. V. K, C. Sabu, N. Kalarikkal, and S. Thomas, "Cellulose nanocomposites: fabrication and biomedical applications," Journal of Bioresources and Bioproducts, vol. 5, no. 4 , pp. 223-237, 2020.

[66] R. Miraftab and X. Huining, "Feasibility and potential of graphene and its hybrids with cellulose as drug carriers: a commentary," Journal of Bioresources and Bioproducts, vol. 4, no. 4, pp. 200-201, 2019.

[67] S. Chatterjee and P. Chi-leung Hui, "Review of stimuli-responsive polymers in drug delivery and textile application," Molecules, vol. 24, no. 14, p. 2547, 2019.

[68] M. Akbari, A. Tamayol, V. Laforte et al., "Composite living fibers for creating tissue constructs using textile techniques," Advanced Functional Materials, vol. 24, no. 26, pp. 40604067, 2014.

[69] N. D. Oltarzhevskaya and M. A. Korovina, "Medical textile and hydrogel materials for targeted delivery of drugs in oncological practice," Russian Journal of General Chemistry, vol. 82, no. 13, pp. 2294-2305, 2012.

[70] W. Wang, E. Wat, P. C. Hui et al., "Dual-functional transdermal drug delivery system with controllable drug loading based on thermosensitive poloxamer hydrogel for atopic dermatitis treatment," Scientific Reports, vol. 6, p. 24112, 2016. 